\title{
Sovereign CDS calibration under a hybrid Sovereign Risk Model
}

\author{
Gian Luca De Marchi ${ }^{1} \quad$ Marco Di Francesco ${ }^{2} \quad$ Sidy Diop $^{3} \quad$ Andrea Pascucci $^{4}$
}

This version: March 13, 2019

\begin{abstract}
The European sovereign debt crisis, started in the second half of 2011, has posed the problem for asset managers, trades and risk managers to assess sovereign default risk. In the reduced form framework, it is necessary to understand the interrelationship between creditworthiness of a sovereign, its intensity to default and the correlation with the exchange rate between the bond's currency and the currency in which the CDS spread are quoted. To do this, we propose a hybrid sovereign risk model in which the intensity of default is based on the jump to default extended CEV model. We analyze the differences between the default intensity under the domestic and foreign measure and we compute the default-survival probabilities in the bond's currency measure. We also give an approximation formula to CDS spread obtained by perturbation theory and provide an efficient method to calibrate the model to CDS spread quoted by the market. Finally, we test the model on real market data by several calibration experiments to confirm the robustness of our method.
\end{abstract}

This is an Accepted Manuscript of an article published by Taylor \& Francis in Applied Mathematical Finance on 27 Dec 2018, available online:

http://www.tandfonline.com/10.1080/1350486X.2018.1554447

\section{KEYWORDS}

credit default swap; hybrid credit-equity model; Constant Elasticity of Variance model; asymptotic expansion; Foreign exchange rate;

\section{Introduction}

Recent dynamic of sovereign credit risk in Europe has determined some significant doubts on the paradigm considering a Euro area government bond as a risk free investment. Consequently for investors the

\footnotetext{
${ }^{1}$ UnipolSai Assicurazioni s.p.a., Bologna, Italy. e-mail:gianlucademarchi@unipol.it

2 UnipolSai Assicurazioni s.p.a., Bologna, Italy. e-mail: marco.difrancesco@unipolsai.it

${ }^{3}$ Dipartimento di Matematica, Università di Bologna, Bologna, Italy. e-mail: sidy.diop2@unibo.it

${ }^{4}$ Dipartimento di Matematica, Università di Bologna, Bologna, Italy. e-mail: andrea.pascucci@unibo.it
} 
identification and pricing of sovereign bonds becomes a crucial issue. Main factors determining this structural change are the following:

- lack of a common economic and financial policy, with investors' perception that the economic and political convergence of the Euro area still required a long time;

- target to stabilize government deficits constantly disregarded by governments with the impossibility of financing infrastructural investments and difficulties in reforming the social security system;

- slowdown in economic growth and interdependence between financial sector crisis and sovereign risk for some countries;

- contagion effect triggered by the PSI in Greece and hence extended to the entire Euro system (aggravated by the downgrading of rating agencies) with a consequent increase in the risk premium requested by investors.

In the second half of 2011, following the escalation of the sovereign debt crisis in the Euro area and the contagion of tensions from the peripheral countries (Greece, Ireland and Portugal) to the core countries of the euro area, foreign demand for eurozone debt has suffered a major collapse involving the liquidation of outstanding positions in particular by institutional investors. The central element that led to this substantial change in terms of asset allocation was the perception that only a few eurozone countries could be considered risk free; in addition, there was the growing fear of the Euro break-up which helped to stimulate the dynamics of cross-border capital outflows.

During the period July - October 2011, foreign investors sold Eurozone fixed income instruments for around 88 bln euro against a 320 bln euro inflow in the first half of 2011. Japanese investors sold almost $98 \%$ of the Greek bonds and $61 \%$ of the Portuguese bonds; in the same period the sale of Italian bonds was almost 10.5\%. Starting from Lehman default event (September 2008), government bonds spreads have suffered a dramatic widening phase both in countries with weak public sector finance and in countries considered to be safer. The volatility of the government bonds spread seems to reflect not only the perceived default risk of the issuers but also some other new relevant factors:

- Aggregate risk (change in monetary policy, global uncertainty, risk aversion);

- Liquidity risk;

- Country specific risk;

- Contagion and systematic risk;

- Exchange rate risk.

The lack of models for the assessment of component represented in the spread risk (eg Break up Euro scenario) and the lack of measures deemed sufficiently robust to quantify sovereign risk have led most investors to a hyper-prudent assessment of the situation, based on worst case hypotheses, negatively distorting the dynamics of spreads. 


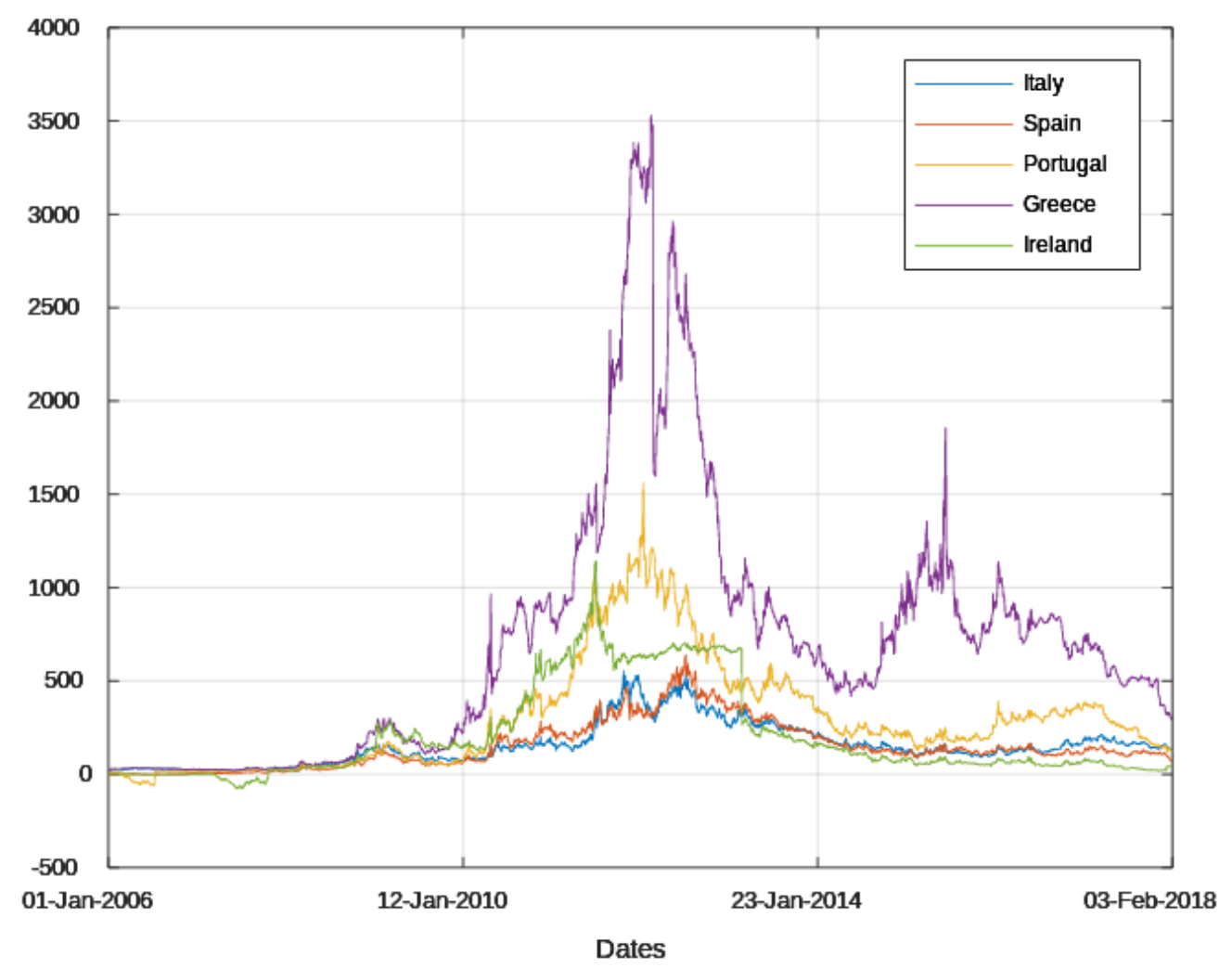

Figure 1. Government Bonds Spread versus Bund 
Default statistics currently used to calibrate corporate credit ratings are not applicable to sovereign risk. Furthermore, as reported in Moody's Investor Service Sovereign Default and Recovery Rates study [14], there are very limited number of developed countries default events in the last 30 years (Greece in March 2012 and December 2012, Cyprus in July 2013) and consequently it is not possible to infer a consistent rating migration rates matrix for those countries. Also statistics on recovery rates available on defaulted sovereign bonds are estimated mainly with reference to emerging countries; the average recovery rates reported by Moody's in the sovereign default study is higher than the recovery rates for the two defaults of Greece in 2012 and for the one of Cyprus in 2013.

The need for banks and financial institutions to assess the risk associated with government bonds exposures has posed the problem for asset managers, traders and risk managers to determine how to assess sovereign default risk. There is no a specific standard in models used to assess the sovereign default risk and practitioners make use of consolidated models developed for corporate bonds. The two main families of models used to price and assess the risk of corporate and sovereign bonds are reduced-form models and structural models. Whereas reduced-form models are based on the specification of the risk-neutral default intensity and the fractional loss model, the structural models focus on the behavior of the assets of the issuer and the relative volatility compared to the value of the liabilities. Structural models have varied widely in their implementation, starting from the original models developed by Black and Scholes (1973) and Merton (1974) and moving to more complex specifications making assumptions concerning the capital structures of the issuers and including different types of debts and other form of liabilities. While in structural models the default time is usually a predictable stopping time, defined as the first hitting time to a certain barrier by the asset process, in the reduce form the default time is a totally unpredictable stopping time modeled as the first jump of a Poisson process with stochastic intensity.

In the reduced form models, thanks to one of the fundamental property of jumps in Poisson process, the survival probabilities can be computed as a discount factors, and so it is a common market practice to compute these probabilities from credit default swap market instead from bond market. Moreover, the market of sovereign credit default swaps (SCDS) contracts has grown very fast in the last decade and has become very liquid, clean and standardized. So, the market of SCDS offers a consistent data framework set to estimate the default-survival probabilities. Furthermore estimates retrieved from CDS market prices allow practitioners to exclude the issue to represent the liquidity component of bonds spreads.

In this paper we consider fixed Loss Given Default, that is a standard practice in the market and supported by historical observation. Unlike corporate CDS contracts, SCDS are usually denominated in a difference currency than the currency of the underling bonds. This is due to avoid the risk of depreciation of the bond's currency in case of a credit event. In fact, if SCDS were denominated in the same currency as the bond, the recovery value would be significantly distorted by exchange rate fluctuation. So, for example, the market convention is to trade Euro CDS in US dollar and US CDS in Euro. The different currency between SCDS and bonds market makes impossible to use the usual bootstrap technique to 
compute the default-survival probabilities in the bond's currency measure as for a corporate firm. Moreover, the assumption that the foreign and domestic hazard rate are identical is not realistic and contradicts market observations. So, the joint evolution of the domestic hazard rate and the FX rate between the two currencies must be modeled.

One of the motivation of this work has been to better understand the interrelationship between the creditworthiness of a sovereign, its intensity to default and the exchange rate between its bond's currency and the currency in which SCDS contracts are quoted. We analyze the differences between the default intensity under the domestic and foreign measure and we compute the default-survival probabilities in the bond's currency measure. Finally, we test our calibration to the valuation of sovereign bonds even during the period of sovereign crisis.

We start by providing a robust and efficient method to calibrate a hybrid sovereign risk model to SCDS market. We first present a model for the intensity of default of a sovereign government based on the jump to default extended CEV (Constant Elasticity Variance) model introduced in [3] in 2006 by establishing the link with the exchange rate. Then we give an approximation formula to the SCDS spread obtained from perturbation theory.

Our approach is similar to [2] where the authors presented a model that captures the link between the sovereign default intensity and the foreign exchange rate by adding a constant in case of credit event to this exchange rate process. As shown in [5], the introduction of a jump in the dynamic of FX rate is necessary since a purely diffusion-based correlation between the exchange rate the hazard rate is not able to explain market observations. The default intensity is described by the the exponential of some Ornstein-Uhlenbeck processes. Our paper differs from [2] in several aspects: first we provide a hybrid model that captures the default intensity of the sovereign. Second, to approximate the SCDS spread, we employ a recent methodology introduced in [11, 15], which consists in an asymptotic expansion of the solution to the pricing partial differential equation. This approach of describing the sovereign default intensity with a hybrid model has been introduced in [10]. The authors are also inspired by the JDCEV model [3] which has been originally proposed for assessing corporate credit risks.

This paper is organized as follows. In Section 2 we set the notations and introduce the model. In Section 3 we recall the definitions, properties on SCDS spread and provide an explicit approximation formula. Section 4 contains the numerical test: we calibrate the model to Italian USD-quoted CDS contracts assessing in two different periods: at the outbreak of the government crisis at the end of 2011, in which the Italian CDS spreads reached the maximum, and at the present date. In Appendix, to show the robustness and the accuracy of our method, we present other several calibration tests, at the same dates as for Italian USD-quoted CDS spreads, for other European sovereign CDS spreads (France, Spain, Portugal). 


\section{Model and Set-up}

In this section, we follow the approach in [10] to capture the dynamics of the default intensity by considering an hybrid model. This approach is inspired by the work [3] introduced in 2006 and establishes the dependency of the default intensity of the sovereign to its solvency. This latter is an indicator taking into account macro-economical factors like the public debt of the GDP (Gross Domestic Product) ratio, the surplus to GDP, interest rate on the sovereign bonds, GDP growth rate, etc... In what follows we model this solvency by a continuous-time process $S$. Consider the filtered probability space $(\Omega, \mathcal{G}, \mathbb{G}, \mathbb{Q})$ with finite time horizon $T<\infty$. The filtration $\mathbb{G}=\left(\mathcal{G}_{t}\right)_{t \in[0, T]}$ is assumed to satisfy the usual conditions, $\mathcal{G}_{T}=\mathcal{G}$ and is generated by the Brownian motions $W_{t}^{1}$ and $W_{t}^{2}$ and some discontinuous stochastic process $D_{t}$. Let $\varepsilon$ be an exponentially distributed random variable with parameter 1 (i.e. $\varepsilon \sim \operatorname{Exp}(1)$ ).

Let $X$ be a stochastic process defined as

$$
\mathrm{d} X_{t}=\left(r_{d}(t)-\frac{1}{2} \sigma^{2}\left(t, X_{t}\right)+\lambda\left(t, X_{t}\right)\right) \mathrm{d} t+\sigma\left(t, X_{t}\right) \mathrm{d} W_{t}^{1},
$$

where $r_{d}$ is deterministic taking values in $\mathbb{R}$ and $\lambda$ is a $\mathbb{G}$-adapted process. We assume that the time- and state-dependent functions $\sigma=\sigma(t, X)$ and $\lambda=\lambda(t, X)$ are positive, differentiable with respect to $X$ and uniformly bounded. Let $L$ be a real positive constant with $L<e^{X_{0}}$. Let $\zeta$ be defined as

$$
\zeta=\inf \left\{t>0 \mid e^{X_{t}} \leq L\right\} \wedge \inf \left\{t \geq 0 \mid \int_{0}^{t} \lambda\left(s, X_{s}\right) \mathrm{d} s \geq \varepsilon\right\}
$$

By definition, $\zeta$ is $\mathbb{G}$-stopping time.

Assumption 2.1. (1) The market is modelled by the filtered probability space $(\Omega, \mathcal{G}, \mathbb{G}, \mathbb{Q})$ defined above where $\mathbb{Q}$ is a domestic spot risk-neutral martingale measure and $\mathbb{G}$ represents the quantity of informations of the market and to which all processes are adapted.

(2) The time to default of the sovereign is the stopping time $\zeta$ defined in (2.1) and we define the solvency $S$ of the sovereign as follow:

$$
S_{t}=S_{0} e^{X_{t}} 1_{\{\zeta>t\}}, \quad S_{0}=e^{X_{0}} .
$$

Default happens when the solvency becomes worthless in one of these two ways. Either the process $e^{X}$ falls below $L$ via diffusion or a jump-to-default occurs from a value greater than $L$, where $L$ represents a threshold of the sovereign debt crisis. In what follows, we denote by $\mathbb{F}=\left\{\mathcal{F}_{t}, t \geq 0\right\}$ the filtration generated by the sovereign solvency and by $\mathbb{D}=\left\{\mathcal{D}_{t}, t \geq 0\right\}$ the filtration generated by the process $D_{t}=\mathbb{1}_{\{\zeta \leq t\}}$. Eventually, $\mathbb{G}=\left\{\mathcal{G}_{t}, t \geq 0\right\}, \mathcal{G}_{t}=\mathcal{F}_{t} \vee \mathcal{D}_{t}$ is the enlarged filtration.

(3) The rate of exchange between foreign currency $c_{f}$ and domestic currency $c_{d}$ is denoted by $Z_{t} \geq 0$, $r_{i}$ are the short-term interest rates and $B_{i}(t)=e^{\int_{0}^{t} r_{i}(u) \mathrm{d} u}$ the instantaneous bank accounts in the 
respective currencies $c_{i}, i=d, f$.

We assume that the rate of exchange $Z$ satisfies a SDE of the form

$$
\mathrm{d} Z_{t}=\mu_{t}^{Z} Z_{t^{-}} \mathrm{d} t+\eta Z_{t^{-}} \mathrm{d} W_{t}^{2}+\gamma Z_{t^{-}} \mathrm{d} D_{t}, \quad \text { with } \mathrm{d} W_{t}^{1} \mathrm{~d} W_{t}^{2}=\rho \mathrm{d} t
$$

where $\eta>0$ and $\gamma \in[-1,1]$ is the devaluation/revaluation rate of the FX process. The dynamics (2.2) captures the dependency between the sovereign default risk and the rate of exchange, first through the correlation $\rho$ between the Brownian motion $W^{1}$ and $W^{2}$ and then via the coefficient of devaluation/evaluation $\gamma$. Indeed, there is a jump on the rate of exchange at the time of default $\zeta$ by

$$
\Delta Z_{\zeta}=\gamma Z_{\zeta^{-}}
$$

That is at $\zeta$, the foreign currency $c_{f}$ is evaluated/devaluated with respect to the domestic currency $c_{d}$ in a jump fraction $\gamma$ of the pre-default value of $Z$. Therefore the price in $c_{d}$ of the foreign instantaneous bank account at time $t$ is $B_{f}(t) Z_{t}$. By Ito formula and (2.2)

$$
\begin{aligned}
\mathrm{d}\left(B_{f}(t) Z_{t}\right) & =B_{f}(t) \mathrm{d} Z_{t}+r_{f}(t) B_{f}(t) Z_{t} \mathrm{~d} t \\
& =r_{f}(t) B_{f}(t) Z_{t} \mathrm{~d} t+\mu_{t}^{Z} B_{f}(t) Z_{t} \mathrm{~d} t+\eta B_{f}(t) Z_{t} \mathrm{~d} W_{t}^{2}+\gamma B_{f}(t) Z_{t} \mathrm{~d} D_{t} \\
& =B_{f}(t) Z_{t}\left(\left(r_{f}(t)+\mu_{t}^{Z}+\gamma\left(1-D_{t}\right) \lambda\left(t, X_{t}\right)\right) \mathrm{d} t+\eta \mathrm{d} W_{t}^{2}+\gamma \mathrm{d} M_{t}\right)
\end{aligned}
$$

where the process $\mathrm{d} M_{t}=\mathrm{d} D_{t}-\mathrm{d} A_{t}$ is a martingale with $A_{t}=\int_{0}^{t}\left(1-D_{s}\right) \lambda_{s} \mathrm{~d} s$ the compensator of $D_{t}$.

Proposition 2.2. If the rate of exchange between the foreign and domestic currencies obeys a stochastic differential equation (2.2), and if the riskless short-term rates of return in the domestic and foreign currencies are respectively $r_{i}, i=d, f$, then under $\mathbb{Q}$

$$
\mu_{t}^{Z}=r_{d}(t)-r_{f}(t)-\gamma\left(1-D_{t}\right) \lambda\left(t, X_{t}\right)
$$

Therefore, the exchange rate is given by

$$
Z_{t}=Z_{0} \exp \left(\int_{0}^{t}\left(r_{d}(s)-r_{f}(s)-\gamma\left(1-D_{s}\right) \lambda\left(s, X_{s}\right)\right) \mathrm{d} s-\frac{1}{2} \eta^{2} t+\eta W_{t}^{2}+\gamma D_{t}\right)
$$

Proof. Under $\mathbb{Q}$, the discounted value in $c_{d}$ of the foreign bank account must be a martingale. But the dynamics of the discounted value $\frac{B_{f}(t)}{B_{d}(t)} Z_{t}$ at time $t$ is, by equation (2.3)

$$
\begin{aligned}
\mathrm{d}\left(\frac{B_{f}(t)}{B_{d}(t)} Z_{t}\right) & =-r_{d}(t) \frac{B_{f}(t)}{B_{d}(t)} Z_{t} \mathrm{~d} t+\frac{1}{B_{d}(t)} \mathrm{d}\left(B_{f}(t) Z_{t}\right) \\
& =\frac{B_{f}(t)}{B_{d}(t)} Z_{t}\left(\left(r_{f}(t)-r_{d}(t)+\mu_{t}^{Z}+\gamma\left(1-D_{t}\right) \lambda\left(t, X_{t}\right)\right) \mathrm{d} t+\left(\eta \mathrm{d} W_{t}^{2}+\gamma \mathrm{d} M_{t}\right)\right)
\end{aligned}
$$


Since the term $\left(\eta \mathrm{d} W_{t}^{2}+\gamma \mathrm{d} M_{t}\right)$ is a martingale, then we must have

$$
r_{f}(t)-r_{d}(t)+\mu_{t}^{Z}+\gamma\left(1-D_{t}\right) \lambda\left(t, X_{t}\right)=0
$$

Proposition 2.3. Let $\mathbb{Q}_{f}$ be the risk-neutral foreign martingale measure. Then $\mathbb{Q}$ and $\mathbb{Q}_{f}$ are mutually absolutely continuous; that is they are related by the likelihood ratio

$$
\left(\frac{\mathrm{d} \mathbb{Q}_{f}}{\mathrm{dQ}}\right)_{\mathcal{F}_{T}}=\exp \left(\eta W_{T}^{2}+\gamma M_{T}-\frac{1}{2} \eta^{2} T\right)
$$

Proof. Consider a contingent claim whose value at time $t$ inc $c_{f}$ is $V_{t}$. The price $V_{0}$ of the claim at time $t=0$ in $c_{f}$ is the discounted expected value of its price, in $c_{f}$, at time $T$, where the expectation is computed under $\mathbb{Q}_{f}$, the risk-neutral foreign martingale measure:

$$
V_{0}=e^{-\int_{0}^{T} r_{f}(s) \mathrm{d} s} \hat{E}\left[V_{T}\right]
$$

Let $U_{t}$ be the time- $t$ price of the claim in $c_{d}$. Then $U_{t}=V_{t} Z_{t}$, where $Z_{t}$ is the rate of exchange between $c_{f}$ and $c_{d}$. Since the claim is a tradable asset, its price in $c_{d}$ must be a martingale under $\mathbb{Q}$. In particular the time-zero price is the discounted expected value of the time- $T$ price:

$$
\begin{aligned}
U_{0} & =e^{-\int_{0}^{T} r_{d}(s) \mathrm{d} s} E\left[U_{T}\right] \\
V_{0} Z_{0} & =e^{-\int_{0}^{T} r_{d}(s) \mathrm{d} s} E\left[V_{T} Z_{T}\right] \\
V_{0} & =e^{-\int_{0}^{T} r_{f}(s) \mathrm{d} s} E\left[V_{T} \frac{Z_{T}}{Z_{0}} e^{-\int_{0}^{T}\left(r_{f}(s)-r_{d}(s)\right) \mathrm{d} s}\right] .
\end{aligned}
$$

Comparing equations (2.5) and (2.6) shows that

$$
\hat{E}\left[V_{T}\right]=E\left[V_{T} \frac{Z_{T}}{Z_{0}} e^{-\int_{0}^{T}\left(r_{f}(s)-r_{d}(s)\right) \mathrm{d} s}\right] .
$$

Since it holds for any non-negative $\mathcal{G}_{T}$-measurable random variable $V_{T}$, it follows from (2.4) that

$$
\left(\frac{\mathrm{d} \mathbb{Q}_{f}}{\mathrm{dQ}}\right)_{\mathcal{F}_{T}}=\frac{Z_{T}}{Z_{0}} e^{-\int_{0}^{T} r_{f}(s)-r_{d}(s) \mathrm{d} s}=\exp \left(\eta W_{T}^{2}+\gamma M_{T}-\frac{1}{2} \eta^{2} T\right) .
$$

Set $Y_{t}=\log \left(Z_{t}\right)$. By Ito formula, the dynamics of $Y$ is given by

$$
\mathrm{d} Y_{t}=\left(\mu_{t}^{Z}-\frac{1}{2} \eta^{2}\right) \mathrm{d} t+\eta \mathrm{d} W_{t}^{2}+\gamma \mathrm{d} D_{t}
$$




$$
=\left(r_{d}(t)-r_{f}(t)-\frac{1}{2} \eta^{2}-\gamma\left(1-D_{t}\right) \lambda\left(t, X_{t}\right)\right) \mathrm{d} t+\eta \mathrm{d} W_{t}^{2}+\gamma \mathrm{d} D_{t} .
$$

Let the volatility of the solvency be defined as

$$
\sigma(t, X)=a(t) e^{(\beta-1) X}
$$

where $\beta<1$ and $a(t)>0$ are the so-called elasticity parameter and scale function. The intensity of default of the sovereign, expressed as a function of the solvency, is defined as

$$
\lambda(t, X)=b(t)+c \sigma(t, X)^{2}=b(t)+c a(t)^{2} e^{2(\beta-1) X}
$$

where $b(t) \geq 0$ and $c \geq 0$ govern the sensitivity of the default intensity with respect to the solvency. Under $\mathbb{Q}$, it follows that the risk-neutral dynamics of the solvency $S_{t}=\left\{S_{t}, t \geq 0\right\}$ is then given by

$$
\left\{\begin{array}{l}
S_{t}=S_{0} e^{X_{t}}\left(1-D_{t}\right), \quad S_{0}>0, \\
\mathrm{~d} X_{t}=\left(r_{d}(t)-\frac{1}{2} \sigma^{2}\left(t, X_{t}\right)+\lambda\left(t, X_{t}\right)\right) \mathrm{d} t+\sigma\left(t, X_{t}\right) \mathrm{d} W_{t}^{1}, \\
\mathrm{~d} Y_{t}=\left(r_{d}(t)-r_{f}(t)-\frac{1}{2} \eta^{2}-\gamma\left(1-D_{t}\right) \lambda\left(t, X_{t}\right)\right) \mathrm{d} t+\eta \mathrm{d} W_{t}^{2}+\gamma \mathrm{d} D_{t}, \\
\mathrm{~d} D_{t}=\gamma\left(1-D_{t}\right) \lambda\left(t, X_{t}\right) \mathrm{d} t+\mathrm{d} M_{t}, \quad \text { with } M \text { a martingale. } \\
\mathrm{d} W_{t}^{1} \mathrm{~d} W_{t}^{2}=\rho \mathrm{d} t, \\
\zeta=\inf \left\{t>0 \mid e^{X_{t}} \leq L\right\} \wedge \inf \left\{t \geq 0 \mid \int_{0}^{t} \lambda\left(t, X_{t}\right) \geq \varepsilon\right\} .
\end{array}\right.
$$

\section{Sovereign Credit Default Swap spread}

A CDS is an agreement between two parties, called the protection buyer and the protection seller, typically designed to transfer to the protection seller the financial loss that the protection buyer would suffer if a particular default event happened to a third party, called the reference entity. The protection seller delivers a protection payment to the protection buyer at the time of the default event. In exchange the protection buyer makes periodic premium payments at time intervals $\alpha$ at the credit default swap rate up to the default event or the expiry maturity, whichever comes first. The protection payment is the specified percentage $(1-\delta)$ of the CDS notional amount $\mathcal{N}$ (=1 by assumption), called loss-given-default. The valuation problem is to determine the arbitrage-free CDS rate $R$ that makes the present value of the CDS contract equal to zero. This rate equates the present value of the protection payoff to the present value of all the premium payments.

By Sovereign, we understand, from the definition given by the International Swaps and Derivatives Association (ISDA), "any state, potential subdivision or government, or any agency, instrumentality, ministry, department or other authority (including ... central bank) thereof". In this paper, for simplicity, 
we consider sovereign governments. Hence a sovereign Credit Default Swap is a CDS where the reference entity is a government. e.g Eurozone States Members. From ISDA, a credit event in sovereign CDS contracts is induced among others by

- Failure to pay: a sovereign fails to make a payment on its obligations (principle, coupons, etc..) in an amount at least as large as the payment requirement beyond the period allowed;

- Restructuring: a sovereign alters the principle amount, coupon, currency, maturity or the ranking in priority of repayment of an obligation;

- Repudiation/moratorium: a sovereign refuses to honor its obligation and declares a moratorium.

Here we assume that the premium and protection payments are settled in the foreign currency $c_{f}$. Hence their values $P V$ are given by

$$
\begin{aligned}
\mathrm{PV}(\text { Protection leg }) & =\hat{E}\left[e^{-\int_{t}^{\zeta} r_{f}(u) \mathrm{d} u}(1-\delta) \mathbb{1}_{\{\zeta \leq T\}} \mid \mathcal{G}_{t}\right] \\
& =E\left[e^{-\int_{t}^{\zeta} r_{f}(u) \mathrm{d} u}(1-\delta) L_{\zeta} \mathbb{1}_{\{\zeta \leq T\}} \mid \mathcal{G}_{t}\right] \quad \text { (by change of measure) } \\
& =(1-\delta) E\left[\frac{B_{f}(t)}{B_{f}(\zeta)} \frac{B_{f}(\zeta) Z_{\zeta} B_{d}(t)}{B_{f}(t) Z_{t} B_{d}(\zeta)} \mathbb{1}_{\{\zeta \leq T\}} \mid \mathcal{G}_{t}\right] \\
& =(1-\delta) E\left[\frac{Z_{\zeta} B_{d}(t)}{Z_{t} B_{d}(\zeta)} \mathbb{1}_{\{\zeta \leq T\}} \mid \mathcal{G}_{t}\right] \\
& =(1-\delta) E\left[e^{Y_{\zeta}-Y_{t}} e^{-\int_{t}^{\zeta} r_{d}(u) \mathrm{d} u} \mathbb{1}_{\{\zeta \leq T\}} \mid \mathcal{G}_{t}\right] \\
& =\mathbb{1}_{\{\zeta>t\}}(1-\delta) \int_{t}^{T} E\left[e^{Y_{s}-Y_{t}-\int_{t}^{s}\left(r_{d}(u)+\lambda\left(u, X_{u}\right)\right) \mathrm{d} u} \lambda\left(s, X_{s}\right) \mid \mathcal{F}_{t}\right] \mathrm{d} s \\
\operatorname{PV}(\operatorname{Premium~leg}) & =\sum_{i=1}^{M} \hat{E}\left[e^{-\int_{t}^{t_{i}} r_{f}(u) \mathrm{d} u} \frac{T}{M} R_{t} \mathbb{1}_{\left\{\zeta>t_{i}\right\}} \mid \mathcal{G}_{t}\right] \\
& =\sum_{i=1}^{M} E\left[e^{Y_{t_{i}}-Y_{t}} e^{\left.-\int_{t}^{t_{i}} r_{d}(u) \mathrm{d} u \frac{T}{M} R_{t} \mathbb{1}_{\left\{\zeta>t_{i}\right\}} \mid \mathcal{G}_{t}\right]}\right. \\
& =\frac{T}{M} R_{t} \mathbb{1}_{\{\zeta>t\}} \sum_{i=1}^{M} E\left[e^{\left.Y_{t_{i}}-Y_{t}-\int_{t}^{t_{i}}\left(r_{d}(u)+\lambda\left(u, X_{u}\right)\right) \mathrm{d} u \mid \mathcal{F}_{t}\right]}\right.
\end{aligned}
$$

It follows that the CDS spread at time $\mathrm{t}$ is given by

$$
R_{t}=\frac{\mathbb{1}_{\{\zeta>t\}}(1-\delta) \int_{t}^{T} E\left[e^{Y_{s}-Y_{t}-\int_{t}^{s}\left(r_{d}(u)+\lambda\left(u, X_{u}\right)\right) \mathrm{d} u} \lambda\left(s, X_{s}\right) \mid \mathcal{F}_{t}\right] \mathrm{d} s}{\mathbb{1}_{\{\zeta>t\}} \frac{T}{M} \sum_{i=1}^{M} E\left[e^{Y_{t_{i}}-Y_{t}-\int_{t}^{t_{i}}\left(r_{d}(u)+\lambda\left(u, X_{u}\right)\right) \mathrm{d} u} \mid \mathcal{F}_{t}\right]},
$$

and therefore

$$
R \equiv R_{0}=\frac{(1-\delta) \int_{0}^{T} E\left[e^{Y_{s}-Y_{0}-\int_{0}^{s}\left(r_{d}(u)+\lambda\left(u, X_{u}\right)\right) \mathrm{d} u} \lambda\left(s, X_{s}\right)\right] \mathrm{d} s}{\frac{T}{M} \sum_{i=1}^{M} E\left[e^{Y_{t_{i}}-Y_{0}-\int_{0}^{t_{i}}\left(r_{d}(u)+\lambda\left(u, X_{u}\right)\right) \mathrm{d} u}\right]}
$$


We aim to give an explicit approximation formula to SCDS spread (3.1) based on an asymptotic expansion technique introduced in $[11,16]$. We consider the following general backward Cauchy problem

$$
\begin{cases}\left(\partial_{t}+\mathcal{A}\right) u(t, z)=0, & t<T, z \in \mathbb{R}^{d} \\ u(T, z)=h(z), & z \in \mathbb{R}^{d}\end{cases}
$$

where $\mathcal{A}=\mathcal{A}(t, z)$ is a (locally) parabolic differential operator of the form

$$
\mathcal{A}(t, z)=\sum_{|\alpha| \leq 2} a_{\alpha}(t, z) D_{z}^{\alpha}, \quad t \in \mathbb{R}^{+}, z \in \mathbb{R}^{d}
$$

where

$$
\alpha=\left(\alpha_{1}, \ldots, \alpha_{d}\right), \quad|\alpha|=\sum_{i=1}^{d} \alpha_{1}+\ldots+\alpha_{d}, D_{z}^{\alpha}=\partial_{z_{1}}^{\alpha_{1}} \ldots \partial_{z_{d}}^{\alpha_{d}}
$$

In our specific setting, we will consider $\mathcal{A}$ to be the infinitesimal generator of the stochastic processes $(X, r)$ in (2.8), whose precise expression in given in formula (3.3).

Next, we consider the formal expansions $\mathcal{A}=\sum_{n} \mathcal{A}_{n}$ and $u=\sum_{n} u_{n}$, where the $u_{n}$ 's, for $n \geq 0$, are defined recursively by

$$
\begin{cases}\left(\partial_{t}+\mathcal{A}_{0}\right) u_{0}(t, z)=0, & t<T, z \in \mathbb{R}^{d} \\ u_{0}(T, z)=h(z), & z \in \mathbb{R}^{d}\end{cases}
$$

and

$$
\begin{cases}\left(\partial_{t}+\mathcal{A}_{0}\right) u_{n}(t, z)=-\sum_{k=1}^{n} \mathcal{A}_{k} u_{n-k}(t, z), & t<T, z \in \mathbb{R}^{d}, \\ u_{n}(T, z)=0, & z \in \mathbb{R}^{d},\end{cases}
$$

where

$$
\mathcal{A}_{n}=\sum_{|\alpha| \leq 2} a_{\alpha, n}(t, z) D_{z}^{\alpha}
$$

In (3.6), $\left(a_{\alpha, n}\right)_{0 \leq n \leq N}$ is the $N$-th order Taylor expansion of $a_{\alpha}$, in the spatial variables, around a fixed point $\bar{z}$. Notice that the functions $a_{\alpha, 0}$ depend only on $t$ : hence $\mathcal{A}_{0}$ is a heat operator with time-dependent coefficients and can be written in the form

$$
\mathcal{A}_{0}=\frac{1}{2} \sum_{i, j=1}^{d} C_{i j}(t) \partial_{z_{i} z_{j}}+\sum_{i=1}^{d} m_{i}(t) \partial_{z_{i}}+\chi(t),
$$


for some $C=\left(C_{i j}\right)_{i, j \leq d} \in \mathbb{R}^{d \times d}, m=\left(m_{i}\right)_{i \leq d} \in \mathbb{R}^{d}$ and $\gamma \in \mathbb{R}$. By Duhamel's principle, the solution $u_{0}$ to the $\mathrm{PDE}(3.4)$ is

$$
u_{0}(t, z ; T)=e^{\int_{t}^{T} \gamma(s, z) \mathrm{d} s} \int_{\mathbb{R}^{d}} \Gamma_{0}(t, z ; T, \xi) h(\xi) \mathrm{d} \xi, \quad t<T, z \in \mathbb{R}^{d},
$$

where $\Gamma_{0}$ is the $d$-dimensional Gaussian density

$$
\Gamma_{0}(t, z ; T, \xi)=\frac{1}{\sqrt{2 \pi^{d} \operatorname{det} C(t, T)}} \exp \left(-\frac{1}{2}\left\langle C^{-1}(t, T)(\xi-z-m(t, T)),(\xi-z-m(t, T))\right\rangle\right),
$$

with covariance matrix $C(t, T)$ and mean vector $z+m(t, T)$ given by

$$
C(t, T)=\int_{t}^{T} C(s) d s, \quad m(t, T)=\int_{t}^{T} m(s) d s .
$$

It turns out that, for any $n \geq 0, u_{n}$ can be computed explicitly, as the following result shows.

Theorem 3.1. For any $n \geq 1$, the solution $u_{n}$ to the Cauchy problem (3.5) is given by

$$
u_{n}(t, z ; T)=\mathcal{L}_{n}^{z}(t, T) u_{0}(t, z ; T), \quad t<T, z \in \mathbb{R}^{d}
$$

In $(3.7), \mathcal{L}_{n}^{z}(t, T)$ denotes the differential operator acting on the $z$-variable and defined as

$$
\mathcal{L}_{n}^{z}(t, T)=\sum_{h=1}^{n} \int_{t}^{T} \mathrm{~d} s_{1} \int_{s_{1}}^{T} \mathrm{~d} s_{2} \ldots \int_{s_{h-1}}^{T} \mathrm{~d} s_{h} \sum_{i \in I_{n, h}} \mathcal{G}_{i_{1}}^{z}\left(t, s_{1}\right) \ldots \mathcal{G}_{i_{h}}^{z}\left(t, s_{h}\right)
$$

where

$$
I_{n, h}=\left\{i=\left(i_{1}, \ldots, i_{h}\right) \in \mathbb{N}^{h} \mid i_{1}+i_{2}+\ldots+i_{h}=n\right\}
$$

and the operators $\mathcal{G}_{n}^{z}(t, s)$ are defined as

$$
\mathcal{G}_{n}^{z}(t, s)=\sum_{|\alpha| \leq 2} a_{\alpha, n}\left(s, M^{z}(t, s)\right) D_{z}^{\alpha}
$$

with

$$
M^{z}(t, s)=z+m(t, s)+C(t, s) D_{z}
$$

Proof. A complete proof is given in [11].

Under rather general assumptions on $\mathcal{A}$, the following estimate for the approximation error holds:

$$
\left|u(t, z)-u_{N}(t, z ; T)\right| \leq C_{N}(T-t)^{\frac{N+2}{2}}
$$


where $u_{N}(t, z ; T)$ is the $N$-th order approximation in (3.7) and $C_{N}$ is a positive constant dependent on $N$ but not on $T-t$. Formula (3.9) ensures the short-time asymptotic convergence of the approximation $u_{n}$ to the exact solution $u$ of Cauchy problem (3.2). This theoretical result can be proved by adapting the arguments of [15], Theor. 3.1 This leads to an explicit approximation formula for the SCDS spread (3.1).

Theorem 3.2. Let $T$ be the expiry date of the $S C D S$ contract, $K$ be the total number of premium payments and $t_{i}$ be the $i$-th premium payment date, so that $t_{i+1}-t_{i}=\frac{T}{K}$. Under the general dynamics (2.8), there exist sequences of differential operators $\left(\mathcal{L}_{n}^{1,(x, y)}\right)_{n \geq 0}$ and $\left(\mathcal{L}_{n}^{2,(x, y)}\right)_{n \geq 0}$ of the form of (3.8) and acting on $(x, y)$ such that the $N$-th order approximation of the SCDS spread in (3.1) is given by

$$
R_{N}=\frac{(1-\delta) \int_{0}^{T} \sum_{n=0}^{N} \mathcal{L}_{n}^{1,(x, y)}(0, s) u_{0}(0, x, y ; s) \mathrm{d} s}{\frac{T}{K} \sum_{i=1}^{K} \sum_{n=0}^{N} \mathcal{L}_{n}^{2,(x, y)}\left(0, t_{i}\right) v_{0}\left(0, x, y ; t_{i}\right)}
$$

where

$$
\begin{aligned}
& \begin{aligned}
& u_{0}(0, x, y, s)=e^{-\int_{0}^{s}\left(r_{f}(u)+\frac{1}{2} \eta^{2}+\lambda(u, x)\right) \mathrm{d} u} \\
& \cdot\left(b(s)+c a(s)^{2} \exp \left(2(\beta-1)\left(x+\int_{0}^{s}\left(r_{d}(u)+\left(\beta-\frac{3}{2}\right) \sigma(u, x)^{2}+\lambda(u, x)\right) \mathrm{d} u\right)\right)\right)
\end{aligned} \\
& v_{0}(0, x, y ; s)=e^{-\int_{0}^{s}\left(r_{f}(u)+\frac{1}{2} \eta^{2}+2 \lambda(u, x)\right) \mathrm{d} u} .
\end{aligned}
$$

Proof. We see from (3.1) that we have to evaluate expectations of the form

$$
E_{t}(t)\left[e^{Y_{T}-Y_{t}-\int_{t}^{T}\left(r_{d}(u)+\lambda\left(u, X_{u}\right)\right) \mathrm{d} u} h\left(X_{T}, Y_{T}\right)\right] .
$$

They are functions of $t, X_{t}, Y_{t}$ and $D_{t}$. Let us denote its value at time $t$ for $X_{t}=x, Y_{t}=y$ and $D_{t}=d$ as $f(t, x, y, d)$. Set $g(t, x, y, d)$ the value at time $t$ of

$$
E_{t}(t)\left[e^{Y_{T}-\int_{0}^{T}\left(r_{d}(u)+\lambda\left(u, X_{u}\right)\right) \mathrm{d} u} h\left(X_{T}, Y_{T}\right)\right] .
$$

By Ito formula and its martingale property, one can see that

$$
\begin{aligned}
& \partial_{t} f(t, x, y, d)+\left(r_{d}(t)-\frac{1}{2} \sigma(t, x)^{2}+\lambda(t, x)\right) \partial_{x} f(t, x, y, d)+\frac{1}{2} \sigma(t, x)^{2} \partial_{x}^{2} f(t, x, y, d) \\
& +\left(r_{d}(t)-r_{f}(t)-\frac{1}{2} \eta^{2}+\gamma(1-d) \lambda(t, x)\right) \partial_{y} f(t, x, y, d)+\frac{1}{2} \eta^{2} \partial_{y}^{2} f(t, x, y, d)+\rho \eta \sigma(t, x) \partial_{x y} f(t, x, y, d) \\
& +(1-d) \lambda(t, x)(f(t, x, y, 1)-f(t, x, y, 0))+\left(-r_{f}(t)-\frac{1}{2} \eta^{2}-\lambda(t, x)\right) f(t, x, y, d)=0 .
\end{aligned}
$$

Set

$$
u(t, x, y)=f(t, x, y, 1) \text { and } v(t, x, y)=f(t, x, y, 0)
$$




$$
\Rightarrow f(t, x, y, d)=1_{\{d=1\}} u(t, x, y)+1_{\{d=0\}} v(t, x, y)
$$

For the premium leg, the final conditions of $u$ and $v$ are

$$
\begin{aligned}
& u(T, x, y)=f(T, x, y, 1)=0 \\
& v(T, x, y)=f(T, x, y, 0)=h(x, y)=1 .
\end{aligned}
$$

$u$ is solution to the PDE

$$
\begin{aligned}
& \left(\partial_{t}+\left(r_{d}(t)-\frac{1}{2} \sigma(t, x)^{2}+\lambda(t, x)\right) \partial_{x}+\frac{1}{2} \sigma(t, x)^{2} \partial_{x}^{2}+\left(r_{d}(t)-r_{f}(t)-\frac{1}{2} \eta^{2}\right) \partial_{y}+\right. \\
& \left.+\frac{1}{2} \eta^{2} \partial_{y}^{2}+\rho \eta \sigma(t, x) \partial_{x y}+\left(-r_{f}(t)-\frac{1}{2} \eta^{2}-\lambda(t, x)\right)\right) u=0, \quad \text { for } t<T \text { and } x, y \in \mathbb{R}^{2} \\
& u(T, x, y)=0 \quad x, y \in \mathbb{R}^{2} .
\end{aligned}
$$

It follows that $u \equiv 0$. Therefore, for computing the premium leg, one only need to solve directly the PDE for $v$

$$
\begin{aligned}
& \left(\partial_{t}+\left(r_{d}(t)-\frac{1}{2} \sigma(t, x)^{2}+\lambda(t, x)\right) \partial_{x}+\frac{1}{2} \sigma(t, x)^{2} \partial_{x}^{2}+\left(r_{d}(t)-r_{f}(t)-\frac{1}{2} \eta^{2}+\gamma \lambda(t, x)\right) \partial_{y}+\right. \\
& \left.+\frac{1}{2} \eta^{2} \partial_{y}^{2}+\rho \eta \sigma(t, x) \partial_{x y}+\left(-r_{f}(t)-\frac{1}{2} \eta^{2}-2 \lambda(t, x)\right)\right) v=0, \quad \text { for } t<T \text { and } x, y \in \mathbb{R}^{2} \\
& v(T, x, y)=1 \quad x, y \in \mathbb{R}^{2} .
\end{aligned}
$$

Analogously, to compute the protection leg, one solve the following PDE for $\mathrm{u}$

$$
\begin{aligned}
& \left(\partial_{t}+\left(r_{d}(t)-\frac{1}{2} \sigma(t, x)^{2}+\lambda(t, x)\right) \partial_{x}+\frac{1}{2} \sigma(t, x)^{2} \partial_{x}^{2}+\left(r_{d}(t)-r_{f}(t)-\frac{1}{2} \eta^{2}\right) \partial_{y}+\right. \\
& \left.+\frac{1}{2} \eta^{2} \partial_{y}^{2}+\rho \eta \sigma(t, x) \partial_{x y}+\left(-r_{f}(t)-\frac{1}{2} \eta^{2}-\lambda(t, x)\right)\right) u=0, \quad \text { for } t<T \text { and } x, y \in \mathbb{R}^{2} \\
& u(T, x, y)=\lambda(T, x) \quad x, y \in \mathbb{R}^{2} .
\end{aligned}
$$

Hence to approximate the Quanto CDS spread, we must deal with two Cauchy problems with different operator $\mathcal{A}^{1}$ and $\mathcal{A}^{2}$ and different terminal conditions:

$$
\begin{cases}\left(\partial_{t}+\mathcal{A}^{1}\right) u(t, x, y)=0, & t<T, x, y \in \mathbb{R} \\ u(T, x, y)=\lambda(T, x), & x, y \in \mathbb{R},\end{cases}
$$


and

$$
\begin{cases}\left(\partial_{t}+\mathcal{A}^{2}\right) v(t, x, y)=0, & t<T, x, y \in \mathbb{R} \\ v(T, x, y)=1, & x, y \in \mathbb{R},\end{cases}
$$

where

$$
\begin{gathered}
\mathcal{A}^{1}=\frac{1}{2} \sigma(t, x)^{2} \partial_{x}^{2}+\rho \eta \sigma(t, x) \partial_{x y}+\frac{1}{2} \eta^{2} \partial_{y}^{2}+\left(r_{d}(t)-\frac{1}{2} \sigma(t, x)^{2}+\lambda(t, x)\right) \partial_{x} \\
+\left(r_{d}(t)-r_{f}(t)-\frac{1}{2} \eta^{2}\right) \partial_{y}-\left(r_{f}(t)+\frac{1}{2} \eta^{2}+\lambda(t, x)\right)
\end{gathered}
$$

and

$$
\begin{gathered}
\mathcal{A}^{2}=\frac{1}{2} \sigma(t, x)^{2} \partial_{x}^{2}+\rho \eta \sigma(t, x) \partial_{x y}+\frac{1}{2} \eta^{2} \partial_{y}^{2}+\left(r_{d}(t)-\frac{1}{2} \sigma(t, x)^{2}+\lambda(t, x)\right) \partial_{x} \\
+\left(r_{d}(t)-r_{f}(t)-\frac{1}{2} \eta^{2}+\gamma \lambda(t, x)\right) \partial_{y}-\left(r_{f}(t)+\frac{1}{2} \eta^{2}+2 \lambda(t, x)\right)
\end{gathered}
$$

Hence by theorem 3.1, there exists a sequence $\left(\mathcal{L}_{n}^{1,(x, y)}\right)_{b \geq n}$ of differential operators such that the N-th approximation of the solution $u$ of (3.11) is given by

$$
u(0, x, y ; s)=\sum_{n=0}^{N} \mathcal{L}_{n}^{1,(x, y)}(0, s) u_{0}(0, x, y ; s),
$$

where

$$
u_{0}(0, x, y ; s)=e^{\int_{0}^{s} \chi(u, x, y) \mathrm{d} u} \int_{\mathbb{R}^{2}} \Gamma_{0}\left(0, x, y ; s, \xi_{1}, \xi_{2}\right) \lambda\left(s, \xi_{1}\right) \mathrm{d} \xi_{1} \mathrm{~d} \xi_{2},
$$

with

$$
\chi(u, x, y)=-\left(r_{f}(u)+\frac{1}{2} \eta^{2}+\lambda(u, x)\right)
$$

and $\Gamma_{0}$ is the probability density of the 2-dimensional Gaussian random variable $\left(\Xi_{1}, \Xi_{2}\right)$ with covariance and mean respectively given by

$$
C(0, x, y ; s)=\left(\begin{array}{cc}
\int_{0}^{s} \sigma(u, x)^{2} \mathrm{~d} u & \rho \eta \int_{0}^{s} \sigma(u, x) \mathrm{d} u \\
\rho \eta \int_{0}^{s} \sigma(u, x) \mathrm{d} u & \eta^{2} * s
\end{array}\right)
$$

and

$$
m(0, x, y ; s)=\left(x+\int_{0}^{s}\left(r_{d}(u)-\frac{1}{2} \sigma(u, x)^{2}+\lambda(u, x)\right) \mathrm{d} u, \quad y+\int_{0}^{s}\left(r_{d}(u)-r_{f}(u)-\frac{1}{2} \eta^{2}\right) \mathrm{d} u .\right)
$$




$$
\int_{\mathbb{R}^{2}} \Gamma_{0}\left(0, x, y ; s, \xi_{1}, \xi_{2}\right) \lambda\left(s, \xi_{1}\right) \mathrm{d} \xi_{1} \mathrm{~d} \xi_{2}=\int_{\mathbb{R}} \bar{\Gamma}_{0}\left(0, x ; s, \xi_{1}\right) \lambda\left(s, \xi_{1}\right) \mathrm{d} \xi_{1},
$$

where $\bar{\Gamma}_{0}$ is the marginal probability density function of the random Gaussian random variable $\Xi_{1}$ with variance and mean

$$
\bar{C}(0, x, y ; s)=\int_{0}^{s} \sigma(u, x)^{2} \mathrm{~d} u
$$

and

$$
\bar{m}(0, x, y ; s)=x+\int_{0}^{s}\left(r_{d}(u)-\frac{1}{2} \sigma(u, x)^{2}+\lambda(u, x)\right) \mathrm{d} u
$$

It follows

$$
\begin{aligned}
\int_{\mathbb{R}} \bar{\Gamma}_{0}\left(0, x ; s, \xi_{1}\right) \lambda\left(s, \xi_{1}\right) \mathrm{d} \xi_{1} & =b(s)+c a(s) \int_{\mathbb{R}} \bar{\Gamma}_{0}\left(0, x ; s, \xi_{1}\right) e^{2(\beta-1) \xi_{1}} \mathrm{~d} \xi_{1} \\
& =b(s)+c a(s) E[\Pi]
\end{aligned}
$$

where $\Pi=e^{2(\beta-1) \Xi_{1}}$ is a log-normal distributed random variable with mean $E[\Pi]$ equals to

$$
\begin{aligned}
E[\Pi] & =\exp \left(2(\beta-1) \bar{m}(0, x, y ; s)+4(\beta-1)^{2} \frac{\bar{C}(0, x, y ; s)}{2}\right) \\
& =\exp (2(\beta-1)(\bar{m}(0, x, y ; s)+(\beta-1) \bar{C}(0, x, y ; s))) \\
& =\exp \left(2(\beta-1)\left(x+\int_{0}^{s}\left(r_{d}(u)-\frac{1}{2} \sigma(u, x)^{2}+\lambda(u, x)\right) \mathrm{d} u+(\beta-1) \int_{0}^{s} \sigma(u, x)^{2} \mathrm{~d} u\right)\right) \\
& =\exp \left(2(\beta-1)\left(x+\int_{0}^{s}\left(r_{d}(u)+\left(\beta-\frac{3}{2}\right) \sigma(u, x)^{2}+\lambda(u, x)\right) \mathrm{d} u\right)\right) .
\end{aligned}
$$

Hence

$$
\begin{aligned}
u_{0}(0, x, y, s)= & e^{-\int_{0}^{s}\left(r_{f}(u)+\frac{1}{2} \eta^{2}+\lambda(u, x)\right) \mathrm{d} u} \times \\
& \times\left(b(s)+c a(s)^{2} \exp \left(2(\beta-1)\left(x+\int_{0}^{s}\left(r_{d}(u)+\left(\beta-\frac{3}{2}\right) \sigma(u, x)^{2}+\lambda(u, x)\right) \mathrm{d} u\right)\right)\right) .
\end{aligned}
$$

Analogously, we see from theorem 3.1 that there exists a sequence $\left(\mathcal{L}_{n}^{2,(x, y)}\right)_{b \geq n}$ of differential operators such that the N-th approximation of the solution $v$ of (3.12) is given by

$$
v(0, x, y ; s)=\sum_{n=0}^{N} \mathcal{L}_{n}^{2,(x, y)}(0, s) v_{0}(0, x, y ; s),
$$


where

$$
v_{0}(0, x, y ; s)=e^{\int_{0}^{s} \chi(u, x, y) \mathrm{d} u} \int_{\mathbb{R}^{2}} \Gamma_{0}\left(0, x, y ; s, \xi_{1}, \xi_{2}\right) \mathrm{d} \xi_{1} \mathrm{~d} \xi_{2}=e^{\int_{0}^{s} \chi(u, x, y) \mathrm{d} u}
$$

with

$$
\chi(u, x, y)=-\left(r_{f}(u)+\frac{1}{2} \eta^{2}+2 \lambda(u, x)\right)
$$

\section{Sovereign CDS calibration and empirical test}

In this section we apply the method developed in Section 3 to calibrate the model (2.8) to the sovereign CDS spreads quoted by the market. We use quotation for Italian USD-quoted CDS provided by Bloomberg database on the date November, $15^{t h} 2011$ in order to check the robustness of our methodology. We use the second-order approximation formula (3.10) for the SCDS. We consider SCDS contracts with maturity from one up to four years and paid quarterly with recovery rate $40 \%$ at the event of default.

Since the formula (3.10) gives the approximation of the SCDS spread in the domestic currency $c_{d}$ (EUR) and the market SCDS is in the foreign currency $c_{f}$ (USD), we consider the following formula introduced in [2]:

$$
R=\frac{\widetilde{R}}{1+\gamma}
$$

where $\widetilde{R}$ is SCDS spread in the USD. This shows the link between the SCDS spreads in two currencies. To add more flexibility to the model, we assume that the coefficients $a(t)$ and $b(t)$ in (2.7) are linearly dependent on time: more precisely, we assume that

$$
a(t)=a_{1} t+a_{2}, \quad b(t)=b_{1} t+b_{2},
$$

for some constants $a_{1}, a_{2}, b_{1}$, and $b_{2}$. The problem of calibrating the model (2.8) is formulated as an optimization problem. We want to minimize the error between the model CDS spread and the market CDS spreads. Our approach is to use the square difference between market and model CDS spreads. This leads to the nonlinear least square method

$$
\inf _{\Theta} F(\Theta), \quad F(\Theta)=\sum_{i=1}^{N} \omega_{i}\left|R_{i}-\widehat{R}_{i}\right|^{2},
$$

where $N$ is the number of spreads used, $\omega_{i}$ is a weight, $\widehat{R}_{i}$ is the market CDS spreads of the considered 
reference entity observed at time $t=0$ and $\Theta=\left(a_{1}, a_{2}, b_{1}, b_{2}, \beta, c, \eta, \rho, \gamma\right)$, with

$$
a_{2} \geq 0, a_{1} \geq-\frac{a_{2}}{T}, b_{2} \geq 0, b_{1} \geq-\frac{b_{2}}{T}, c \geq 0, \eta \geq 0, \beta<1,-1<\gamma<1 \text { and }-1<\rho<1 .
$$

For the calibration, we use a global optimizer, NMinimize, from Mathematica's optimization toolbox on a PC with $1 \times$ Intel i7-6599U $2.50 \mathrm{GHz}$ CPU and 8GB RAM. First we calibrate the model to real market SCDS spreads taken at the outbreak of the government crisis at the end of 2011. Table 1 shows the results of the calibration to Italian SCDS contracts settled in USD and we can observe that the model gives very good fit to the real market data with a computational time equals to 45.808 seconds. We calibrate our model to market data quoted at November, 15th, 2011, when the Italian CDS spreads reached their maximum value.

Table 1. Calibration to Italy USD CDS quoted as COB November, 15th, 2011

\begin{tabular}{|c|c|c|c|}
\hline Times to maturity (Year) & Market spreads (bps) & Model spreads (bps) & Rel. errors \\
\hline 1.25 & 639.604 & 641.307 & $0.266268 \%$ \\
1.5 & 634.042 & 627.823 & $-0.98089 \%$ \\
1.75 & 617.96 & 615.888 & $-0.335402 \%$ \\
2. & 601.52 & 605.829 & $0.71629 \%$ \\
2.25 & 592.68 & 597.979 & $0.894177 \%$ \\
2.5 & 590.589 & 592.642 & $0.347765 \%$ \\
2.75 & 592.193 & 590.018 & $-0.367292 \%$ \\
3. & 594.44 & 590.072 & $-0.734818 \%$ \\
3.25 & 594.935 & 592.299 & $-0.442919 \%$ \\
3.5 & 593.917 & 595.317 & $0.235591 \%$ \\
3.75 & 592.288 & 596.172 & $0.655759 \%$ \\
4. & 590.945 & 589.227 & $-0.290722 \%$ \\
\hline
\end{tabular}

$a_{1}=0.5, a_{2}=0.2, \beta=-0.77, b_{1}=-0.014, b_{2}=0.056, c=0.015, \eta=0.94 \rho=$ $-0.41, \gamma=1.0,45.808$ seconds

We follow the same process as above but this time we calibrate to Italian SCDS spreads quoted on May, 05th 2017. Table 2 shows that the method still provides very good fit to real market data. 
Table 2. Calibration to Italy USD CDS quoted as COB May, 30th 2017

\begin{tabular}{|c|c|c|c|}
\hline Times to maturity (Year) & Market spreads (bps) & Model spreads (bps) & Rel. errors \\
\hline 1.25 & 77.3576 & 78.0834 & $0.938368 \%$ \\
1.5 & 88.3681 & 87.4887 & $-0.995104 \%$ \\
1.75 & 97.1146 & 96.3335 & $-0.804301 \%$ \\
2. & 104.695 & 104.63 & $-0.0616158 \%$ \\
2.25 & 112.006 & 112.395 & $0.346509 \%$ \\
2.5 & 119.142 & 119.642 & $0.419618 \%$ \\
2.75 & 125.994 & 126.39 & $0.314503 \%$ \\
3. & 132.455 & 132.658 & $0.15294 \%$ \\
3.25 & 138.435 & 138.46 & $0.0180013 \%$ \\
3.5 & 143.918 & 143.811 & $-0.0747112 \%$ \\
3.75 & 148.906 & 148.714 & $-0.128773 \%$ \\
4. & 153.4 & 153.161 & $-0.15609 \%$ \\
\hline
\end{tabular}

$a_{1}=0.2, a_{2}=0.03, \beta=0.63, b_{1}=0.01, b_{2}=0.002, c=0.005, \eta=1.068, \rho=$ $0.24, \gamma=0.54,41.992$ seconds

To show the accuracy of the method, we present in the Appendix 5 further calibration tests of the model on SCDS of sovereigns belonging to Eurozone (see 5.2). In particular, we consider the same dates used for the calibration tests to Italian CDS spreads, and we calibrate our model to French, Spanish and Portuguese USD-quoted CDS spreads.

\section{Appendix}

\subsection{Hazard processes and filtration enlargement}

We collect some results on hazard rate and conditional expectation with respect to enlarged filtrations. We present the key formula which relates the conditional expectation with respect to a "big" filtration to the conditional expectation with respect to a "small" filtration. For more about filtration enlargement, we refer for instance to [9].

Let $\zeta$ be a non-negative random variable on a probability space $(\Omega, \mathcal{G}, \mathbb{Q})$, such that $\mathbb{Q}(\zeta=0)=0$ and $\mathbb{Q}(\zeta>t)>0$ for any $t \geq 0$. We introduce a right-continuous process $D$ defined as $D_{t}=\mathbb{1}_{\{\zeta \leq t\}}$, and we denote by $\mathbb{D}$ the filtration generated by $D$; that is $\mathcal{D}_{t}=\sigma\left(D_{u} \mid u \leq t\right)$. Let $\mathbb{F}=\left(\mathcal{F}_{t}\right)_{t \geq 0}$ be a given filtration on $(\Omega, \mathcal{G}, \mathbb{Q})$ such that $\mathbb{G}:=\mathbb{D} \vee \mathbb{F}$; that is we set $\mathcal{G}_{t}:=\mathcal{D}_{t} \vee \mathcal{F}_{t}$ for every $t \in \mathbb{R}_{+}$. Since $\mathcal{D}_{t} \subseteq \mathcal{G}_{t}$ for any $t$, the random variable $\zeta$ is a stopping time with respect to $\mathbb{G}$. The financial interpretation is that the filtration $\mathbb{F}$ models the flow of observations available to the investors prior to the default time $\zeta$. For any $t \in \mathbb{R}_{+}$, we write $F_{t}=\mathbb{Q}\left(\zeta \leq t \mid \mathcal{F}_{t}\right)$, so that $1-F_{t}=\mathbb{Q}\left(\zeta>t \mid \mathcal{F}_{t}\right)$ : notice that $F$ is a bounded and non-negative $\mathbb{F}$-submartingale. We may thus deal with its right-continuous modification.

Definition 5.1. The $\mathbb{F}$-hazard process of $\zeta$, denoted by $\Gamma$, is defined through the formula $1-F_{t}=e^{-\Gamma_{t}}$ for every $t \in \mathbb{R}_{+}$. 
Lemma 5.2. We have $\mathcal{G}_{t} \subset \mathcal{G}_{t}^{*}$, where

$$
\mathcal{G}_{t}^{*}:=\left\{A \in \mathcal{G} \mid \exists B \in \mathcal{F}_{t} \quad A \cap\{\zeta>t\}=B \cap\{\zeta>t\}\right\}
$$

Proof. Observe that $\mathcal{G}_{t}=\mathcal{D}_{t} \vee \mathcal{F}_{t}=\sigma\left(\mathcal{D}_{t}, \mathcal{F}_{t}\right)=\sigma\left(\{\zeta \leq u\}, u \leq t, \mathcal{F}_{t}\right)$. Also, it is easily seen that the the class $\mathcal{G}_{t}^{*}$ is a sub- $\sigma$-field of $\mathcal{G}$. Therefore, it is enough to check that if either $A=\{\zeta \leq u\}$ for $u \leq t$ or $A \in \mathcal{F}_{t}$, then there exists an event $B \in \mathcal{F}_{t}$ such that $A \cap\{\zeta>t\}=B \cap\{\zeta>t\}$. Indeed, in the former case we may take $B=\emptyset$, in the latter $B=A$.

Lemma 5.3. For any $\mathcal{G}$-measurable random variable $Y$ we have, for any $t \in \mathbb{R}_{+}$

$$
E\left[\mathbb{1}_{\{\zeta>t\}} Y \mid \mathcal{G}_{t}\right]=\mathbb{1}_{\{\zeta>t\}} \frac{E\left[Y \mid \mathcal{F}_{t}\right]}{\mathbb{Q}\left(\zeta>t \mid \mathcal{F}_{t}\right)}=\mathbb{1}_{\{\zeta>t\}} e^{\Gamma_{t}} E\left[\mathbb{1}_{\{\zeta>t\}} Y \mid \mathcal{F}_{t}\right] .
$$

Proof. Let us fix $t \in \mathbb{R}_{+}$. In view of the Lemma 5.2. any $\mathcal{G}_{t}$-measurable random variable coincides on the set $\{\zeta>t\}$ with some $\mathcal{F}_{t}$-measurable random variable. Therefore

$$
E\left[\mathbb{1}_{\{\zeta>t\}} Y \mid \mathcal{G}_{t}\right]=\mathbb{1}_{\{\zeta>t\}} E\left[Y \mid \mathcal{G}_{t}\right]=\mathbb{1}_{\{\zeta>t\}} X
$$

where $X$ is an $\mathcal{F}_{t}$-measurable random variable. Taking the conditional expectation with respect to $\mathcal{F}_{t}$, we obtain

$$
E\left[\mathbb{1}_{\{\zeta>t\}} Y \mid \mathcal{F}_{t}\right]=\mathbb{Q}\left(\zeta>t \mid \mathcal{F}_{t}\right) X
$$

Proposition 5.4. Let $Z$ be a bounded $\mathbb{F}$-predictable process. Then for any $t<s \leq \infty$

$$
E\left[\mathbb{1}_{\{t<\zeta \leq s\}} Z_{\zeta} \mid \mathcal{G}_{t}\right]=\mathbb{1}_{\{\zeta>t\}} e^{\Gamma_{t}} E\left[\int_{] t, s]} Z_{u} d F_{u} \mid \mathcal{F}_{t}\right] .
$$

Proof. We start by assuming that $Z$ is a piecewise constant $\mathbb{F}$-predictable process, so that (we are interested only in values of $Z$ for $u \in] t, s]$ )

$$
Z_{u}=\sum_{i=0}^{n} Z_{t_{i}} \mathbb{1}_{] t_{i}, t_{i+1}\right]}(u)
$$

where $t=t_{0}<\ldots<t_{n+1}=s$ and the random variable $Z_{t_{i}}$ is $\mathcal{F}_{t_{i}}$-measurable. In the view of (5.1), for any $i$ we have

$$
\begin{aligned}
E\left[\mathbb{1}_{\left\{t_{i}<\zeta \leq t_{i+1}\right\}} Z_{\zeta} \mid \mathcal{G}_{t}\right] & =\mathbb{1}_{\{\zeta>t\}} e^{\Gamma_{t}} E\left[\mathbb{1}_{\left\{t_{i}<\zeta \leq t_{i+1}\right\}} Z_{t_{i}} \mid \mathcal{F}_{t}\right] \\
& =\mathbb{1}_{\{\zeta>t\}} e^{\Gamma_{t}} E\left[Z_{t_{i}}\left(F_{t_{i+1}}-F_{t_{i}}\right) \mid \mathcal{F}_{t}\right] .
\end{aligned}
$$


In the second step we approximate an arbitrary bounded $\mathbb{F}$-predictable process by a sequence of piecewise constant $\mathbb{F}$-predictable process.

Corollary 5.5. Let $Y$ be a $\mathcal{G}$-measurable random variable. Then, for any $t \leq s$, we have

$$
E\left[\mathbb{1}_{\{\zeta>s\}} Y \mid \mathcal{G}_{t}\right]=\mathbb{1}_{\{\zeta>t\}} E\left[\mathbb{1}_{\{\zeta>s\}} e^{\Gamma_{t}} Y \mid \mathcal{F}_{t}\right]
$$

Furthermore, for any $F_{s}$-measurable random variable $Y$ we have

$$
E\left[\mathbb{1}_{\{\zeta>s\}} Y \mid \mathcal{G}_{t}\right]=\mathbb{1}_{\{\zeta>t\}} E\left[e^{\Gamma_{t}-\Gamma_{s}} Y \mid \mathcal{F}_{t}\right]
$$

If $F$ (and thus $\Gamma$ ) is a continuous increasing process then for any $\mathbb{F}$-predictable bounded process $Z$ we have

$$
E\left[\mathbb{1}_{\{t<\zeta \leq s\}} Z_{\zeta} \mid \mathcal{G}_{t}\right]=\mathbb{1}_{\{\zeta>t\}} E\left[\int_{t}^{s} Z_{u} e^{\Gamma_{t}-\Gamma_{u}} \mathrm{~d} \Gamma_{u} \mid \mathcal{F}_{t}\right]
$$

Proof. In view of (5.1), to show that (5.3) holds, it is enough to observe that $\mathbb{1}_{\{\zeta>s\}}=\mathbb{1}_{\{\zeta>t\}} \mathbb{1}_{\{\zeta>s\}}$. Equality (5.4) is a straightforward consequence of (5.3). Formula (5.5) follows from (5.2) since, when $F$ is increasing, $\mathrm{d} F_{u}=e^{-\Gamma_{u}} \mathrm{~d} \Gamma_{u}$.

\subsection{Further calibration tests}

Table 3. Calibration to France USD CDS quoted as COB November, 15th, 2011

\begin{tabular}{|c|c|c|c|}
\hline Times to maturity (Year) & Market spreads (bps) & Model spreads (bps) & Rel. errors \\
\hline 1.25 & 153.882 & 153.479 & $-0.261987 \%$ \\
1.5 & 158.258 & 159.02 & $0.481714 \%$ \\
1.75 & 164.035 & 164.579 & $0.331552 \%$ \\
2. & 170.14 & 170.156 & $0.00938846 \%$ \\
2.25 & 175.829 & 175.751 & $-0.0445177 \%$ \\
2.5 & 181.045 & 181.363 & $0.175564 \%$ \\
2.75 & 186.464 & 186.993 & $0.283517 \%$ \\
3. & 192.24 & 192.64 & $0.208105 \%$ \\
3.25 & 198.586 & 198.304 & $-0.141846 \%$ \\
3.5 & 205.021 & 203.986 & $-0.504832 \%$ \\
3.75 & 211.605 & 209.684 & $-0.907797 \%$ \\
4. & 217.74 & 215.397 & $-1.07585 \%$ \\
4.25 & 223.104 & 221.127 & $-0.886165 \%$ \\
4.5 & 227.526 & 226.872 & $-0.287417 \%$ \\
4.75 & 231.179 & 232.631 & $0.627802 \%$ \\
5. & 233.936 & 238.404 & $1.90985 \%$ \\
\hline
\end{tabular}

$a_{1}=-0.03, a_{2}=0.009, \beta=0.6, b_{1}=-0.004, b_{2}=0.01, c=0.14, \eta=0.08 \rho=$ $-0.6, \gamma=0.84,52.428$ seconds 
Table 4. Calibration to France USD CDS quoted as COB May, 30th 2017

\begin{tabular}{|c|c|c|c|}
\hline Times to maturity (Year) & Market spreads (bps) & Model spreads (bps) & Rel. errors \\
\hline 1.25 & 5.01852 & 4.98324 & $-0.702846 \%$ \\
1.5 & 6.04391 & 6.12876 & $1.40401 \%$ \\
1.75 & 7.28556 & 7.32835 & $0.587229 \%$ \\
2. & 8.64 & 8.58375 & $-0.651094 \%$ \\
2.25 & 9.99688 & 9.89661 & $-1.00297 \%$ \\
2.5 & 11.3472 & 11.2685 & $-0.693951 \%$ \\
2.75 & 12.7118 & 12.7007 & $-0.0867337 \%$ \\
3. & 14.14 & 14.1946 & $0.386329 \%$ \\
3.25 & 15.6773 & 15.7512 & $0.471642 \%$ \\
3.5 & 17.3094 & 17.3713 & $0.357688 \%$ \\
3.75 & 18.9923 & 19.0557 & $0.333576 \%$ \\
4. & 20.775 & 20.8046 & $0.142628 \%$ \\
4.25 & 22.651 & 22.6184 & $-0.144168 \%$ \\
4.5 & 24.5785 & 24.4968 & $-0.332471 \%$ \\
4.75 & 26.4888 & 26.4395 & $-0.185999 \%$ \\
5. & 28.42 & 28.4459 & $0.0910832 \%$ \\
\hline
\end{tabular}

$a_{1}=0.03, a_{2}=0.3, \beta=0.79, b_{1}=0.0003, b_{2}=0.0, c=0.5, \eta=0.4, \rho=0.07, \gamma=$ $0.7,86.056$ seconds

Table 5. Calibration to Portugal USD CDS quoted as COB November, 15th, 2011

\begin{tabular}{|c|c|c|c|}
\hline Times to maturity (Year) & Market spreads (bps) & Model spreads (bps) & Rel. errors \\
\hline 1.25 & 1528.93 & 1543.47 & $0.951086 \%$ \\
1.5 & 1566.41 & 1562.36 & $-0.258228 \%$ \\
1.75 & 1582.78 & 1570.83 & $-0.754834 \%$ \\
2. & 1579.19 & 1567.94 & $-0.712081 \%$ \\
2.25 & 1558.57 & 1553.04 & $-0.354768 \%$ \\
2.5 & 1525.58 & 1525.99 & $0.0268333 \%$ \\
2.75 & 1481.35 & 1487.44 & $0.411513 \%$ \\
3. & 1430.24 & 1439.13 & $0.621161 \%$ \\
3.25 & 1375.66 & 1383.93 & $0.601337 \%$ \\
3.5 & 1322.47 & 1325.79 & $0.251204 \%$ \\
3.75 & 1270.06 & 1269.29 & $-0.060548 \%$ \\
4. & 1223.28 & 1218.89 & $-0.359159 \%$ \\
4.25 & 1184.4 & 1177.92 & $-0.547237 \%$ \\
4.5 & 1153.2 & 1147.57 & $-0.487785 \%$ \\
4.75 & 1126.69 & 1126.17 & $-0.0466888 \%$ \\
5. & 1104.02 & 1109.19 & $0.467984 \%$ \\
\hline
\end{tabular}

$a_{1}=0.012, a_{2}=0.1, \beta=-0.64, b_{1}=0.05, b_{2}=0.1, c=10.7, \eta=0.013 \rho=$ $-0.66, \gamma=0.99,96.196$ seconds 
Table 6. Calibration to Portugal USD CDS quoted as COB May, 30th 2017

\begin{tabular}{|c|c|c|c|}
\hline Times to maturity (Year) & Market spreads (bps) & Model spreads (bps) & Rel. errors \\
\hline 1.25 & 55.7676 & 56.6391 & $1.56276 \%$ \\
1.5 & 67.7586 & 68.2853 & $0.777373 \%$ \\
1.75 & 80.3718 & 79.9073 & $-0.57795 \%$ \\
2. & 92.975 & 91.4484 & $-1.64194 \%$ \\
2.25 & 104.727 & 102.853 & $-1.78851 \%$ \\
2.5 & 115.529 & 114.07 & $-1.2627 \%$ \\
2.75 & 125.543 & 125.049 & $-0.393047 \%$ \\
3. & 135.15 & 135.747 & $0.441821 \%$ \\
3.25 & 144.688 & 146.124 & $0.992659 \%$ \\
3.5 & 154.187 & 156.147 & $1.27133 \%$ \\
3.75 & 163.548 & 165.787 & $1.36936 \%$ \\
4. & 173.19 & 175.025 & $1.05927 \%$ \\
4.25 & 183.136 & 183.842 & $0.385523 \%$ \\
4.5 & 192.933 & 192.231 & $-0.364032 \%$ \\
4.75 & 201.949 & 200.186 & $-0.873256 \%$ \\
5. & 210.07 & 207.707 & $-1.12481 \%$ \\
\hline
\end{tabular}

$a_{1}=0.015, a_{2}=0.5, \beta=0.7, b_{1}=0.0, b_{2}=0.0, c=0.53, \eta=0.78, \rho=-0.65, \gamma=$ $0.7,79.192$ seconds

Table 7. Calibration to Spain USD CDS quoted as COB November, 15th, 2011

\begin{tabular}{|c|c|c|c|}
\hline Times to maturity (Year) & Market spreads (bps) & Model spreads (bps) & Rel. errors \\
\hline 1.25 & 443.089 & 447.5 & $0.995382 \%$ \\
1.5 & 455.942 & 452.898 & $-0.667565 \%$ \\
1.75 & 461.191 & 457.574 & $-0.784297 \%$ \\
2. & 462.81 & 461.585 & $-0.264677 \%$ \\
2.25 & 464.557 & 464.99 & $0.0931244 \%$ \\
2.5 & 466.775 & 467.842 & $0.228544 \%$ \\
2.75 & 469.216 & 470.197 & $0.209013 \%$ \\
3. & 471.4 & 472.106 & $0.149761 \%$ \\
3.25 & 473.03 & 473.626 & $0.125969 \%$ \\
3.5 & 474.222 & 474.819 & $0.125775 \%$ \\
3.75 & 475.314 & 475.759 & $0.0935208 \%$ \\
4. & 476.525 & 476.544 & $0.0040035 \%$ \\
4.25 & 478.011 & 477.313 & $-0.146031 \%$ \\
4.5 & 479.465 & 478.267 & $-0.24985 \%$ \\
4.75 & 480.572 & 479.705 & $-0.180317 \%$ \\
5. & 480.911 & 482.072 & $0.24148 \%$ \\
\hline
\end{tabular}

$a_{1}=-0.06, a_{2}=0.44, \beta=0.52, b_{1}=-0.002, b_{2}=0.044, c=0.4, \eta=0.03 \rho=$ $-0.6, \gamma=0.5,58.548$ seconds 
Table 8. Calibration to Spain USD CDS quoted as COB May, 30th 2017

\begin{tabular}{|c|c|c|c|}
\hline Times to maturity (Year) & Market spreads (bps) & Model spreads (bps) & Rel. errors \\
\hline 1.25 & 33.5073 & 33.4062 & $-0.301704 \%$ \\
1.5 & 36.6139 & 36.5987 & $-0.0413123 \%$ \\
1.75 & 39.678 & 39.7374 & $0.149748 \%$ \\
2. & 42.74 & 42.8222 & $0.192309 \%$ \\
2.25 & 45.7535 & 45.8532 & $0.218024 \%$ \\
2.5 & 48.7076 & 48.8305 & $0.252401 \%$ \\
2.75 & 51.6109 & 51.7541 & $0.277466 \%$ \\
3. & 54.535 & 54.624 & $0.163207 \%$ \\
3.25 & 57.5301 & 57.4403 & $-0.156104 \%$ \\
3.5 & 60.4933 & 60.2031 & $-0.479832 \%$ \\
3.75 & 63.2717 & 62.9123 & $-0.56793 \%$ \\
4. & 65.87 & 65.5682 & $-0.458181 \%$ \\
4.25 & 68.2415 & 68.1708 & $-0.103697 \%$ \\
4.5 & 70.5227 & 70.7201 & $0.279874 \%$ \\
4.75 & 72.8767 & 73.2164 & $0.466098 \%$ \\
5. & 75.59 & 75.6597 & $0.0922325 \%$ \\
\hline
\end{tabular}

$a_{1}=-0.014, a_{2}=0.073, \beta=0.62, b_{1}=0.002, b_{2}=0.001, c=0.28, \eta=0.6, \rho=$ $-0.38, \gamma=0.96,63.304$ seconds

\section{References}

[1] D. Brigo and A. Alfonsi, Credit default swap calibration and derivatives pricing with the SSRD stochastic intensity model, Finance and Stochastics 9 (2005), pp. 29-42.

[2] D. Brigo, N. Pede and A. Petrelli, Multi Currency Credit Default Swaps: Quanto Effects and FX Devaluation Jumps, ArXiv e-prints, 2015.

[3] P. Carr and V. Linetsky, A jump to default extended CEV model: an application of Bessel processes, Finance and Stochastics 10 (2006), pp. 303-330.

[4] D. Duffie, M. Schroder and C. Skiadas, Recursive valuation of defaultable securities and the timing of resolution of uncertainty, The Annals of Applied Probability 6 (1996), pp. 1075-1090.

[5] P. Ehlers and P. Schonbucher, The influence of FX Risk on Credit Spread, Working Paper, ETH, 2016.

[6] R. A. Jarrow and S. M. Turnbull, Pricing Derivatives on Financial Securities Subject to Credit Risk, Journal of Finance 50 (1995), pp. 53-85.

[7] R. A. Jarrow, D. Lando and S. M. Turnbull, A Markov Model for the Term Structure of Credit Risk Spreads, Review of Financial Studies 10 (1997), pp. 481-523.

[8] M. Jeanblanc and M. Rutkowski, Default risk and hazard process, in Mathematical finance - Bachelier Congress, 2000 (Paris), Springer, Berlin, 2002, pp. 281-312. 
[9] M. Jeanblanc, M. Yor and M. Chesney, Mathematical methods for financial market, Springer-Verlag London, Ltd., London, 2009.

[10] Y. Jiao and S. Li, Modelling sovereign risks: from a hybrid model to the generalized density approach, 2015.

[11] M. Lorig, S. Pagliarani and A. Pascucci, Analytical expansions for parabolic equations, SIAM Journal on Applied Mathematics 75 (2015), pp. 468-491.

[12] R. Mendoza-Arriaga, P. Carr and V. Linetsky, Time-changed Markov processes in unified credit-equity modeling, Mathematical Finance. An International Journal of Mathematics, Statistcs and Financial Economics 20 (2010), pp. 527-569.

[13] R. Mendoza-Arriaga and V. Linetsky, Pricing equity default swaps under the jump-to-default extended CEV model, Finance and Stochastics 15 (2011), pp. 513-540.

[14] Moody's Investor Service, Sovereign Default and Recovery Rates, 1983-2016, Data Report, 2017.

[15] S. Pagliarani and A. Pascucci, Asymptotic expansions for degenerate parabolic equations, Comptes Rendus Mathématique. Académie des Sciences. Paris 352 (2014), pp. 1011-1016.

[16] A. Pascucci, PDE and martingale methods in option pricing Vol. 2, Springer, Milan; Bocconi University Press, Milan, 2011. 